\title{
The spectral energy distributions of isolated neutron stars in the resonant cyclotron scattering model
}

\author{
Hao Tong ${ }^{1}$ and Renxin $\mathrm{Xu}^{2}$ \\ ${ }^{1}$ Xinjiang Astronomical Observatory, Chinese Academy of Sciences, School of Physics, \\ Peking University, China \\ email: haotong@ihep.ac.cn \\ ${ }^{2}$ School of Physics and State Key Laboratory of Nuclear Physics and Technology, \\ Peking University, China \\ email: r.x.xu@pku.edu.cn
}

\begin{abstract}
The X-ray dim isolated neutron stars (XDINSs) are peculiar pulsar-like objects, characterized by their very well Planck-like spectrum. In studying their spectral energy distributions, the optical/UV excess is a long standing problem. Recently, Kaplan et al. (2011) have measured the optical/UV excess for all seven sources, which is understandable in the resonant cyclotron scattering (RCS) model previously addressed. The RCS model calculations show that the RCS process can account for the observed optical/UV excess for most sources. The flat spectrum of RX J2143.0+0654 may due to contribution from bremsstrahlung emission of the electron system in addition to the RCS process.
\end{abstract}

